\title{
Correlation between Crystallographic Orientation and Surface Faceting in $\mathrm{UO}_{2}$
}

\author{
Yinbin Miao ${ }^{\mathrm{a}}$, Kun Mo ${ }^{\mathrm{a}, *}$, Tiankai Yao ${ }^{\mathrm{b}}$, Jie Lian $^{\mathrm{b}, * *}$, Jeffrey Fortner ${ }^{\mathrm{a}}$, \\ Laura Jamison $^{\mathrm{a}}$, Ruqing $\mathrm{Xu}^{\mathrm{a}}$, Abdellatif M. Yacout ${ }^{\mathrm{a}}$ \\ ${ }^{a}$ Argonne National Laboratory, Lemont, IL 60439, United States \\ ${ }^{b}$ Rensselaer Polytechnic Institute, Troy, NY 12180, United States
}

\begin{abstract}
Here coordinated experimental efforts to quantitatively correlate crystallographic orientation and surface faceting features in $\mathrm{UO}_{2}$ are reported upon. A sintered polycrystalline $\mathrm{UO}_{2}$ sample was thermally etched to induce the formation of surface faceting features. Synchrotron Laue microdiffraction was used to obtain a precise crystallographic orientation map for the $\mathrm{UO}_{2}$ surface grains. Scanning electron microscopy (SEM) was utilized to collect the detailed information on the surface morphology of the sample. The surface faceting features were found to be highly dependent on the crystallographic orientation. In most cases, Triple-plane structures containing one $\{100\}$ plane and two $\{111\}$ planes were found to dominate the surface of $\mathrm{UO}_{2}$. The orientation-faceting relationship established in this study revealed a practical and efficient method of determining crystallographic orientation based on the surface features captured by SEM images. Keywords: uranium dioxide, surface faceting, morphology, synchrotron diffraction, scanning electron microscopy
\end{abstract}

\section{Introduction}

Surface properties of $\mathrm{UO}_{2}$ play an important role throughout the lifetime of this widely-used commercial nuclear fuel material, from manufacturing to storage. The surface features and corresponding thermodynamic characteristics influence the morphology, size, and distribution

\footnotetext{
*Tel: +1 (630)252-7417. Email: kunmo@anl.gov (K. Mo)

** Tel: +1 (518)276-6081. Email: lianj@rpi.edu (J. Lian)

Preprint submitted to Journal of Nuclear Materials
}

May 27, 2016

(C) 2016. This manuscript version is made available under the Elsevier user license http://www.elsevier.com/open-access/userlicense/1.0/ 
of fission gas bubbles as well as the initiation and propagation of micro-fractures within the nuclear fuel pellets. Hence, a better understanding of the surface behavior of $\mathrm{UO}_{2}$ will help interpret the in-pile behavior of $\mathrm{UO}_{2}$, thereby advancing the capability of precisely predicting fuel performance[1,2,3]. Like many other materials, the surface energy of $\mathrm{UO}_{2}$ varies substantially with crystallographic orientation. This energy variation creates anisotropy in surface energy, which leads to the formation of surface faceting features during annealing. Detailed understanding of these orientation-dependent surface faceting features can not only expand the fundamental knowledge of the surface characteristics of $\mathrm{UO}_{2}$, but can also shine a light on the mechanisms involved in gas swelling and micro-fracture of $\mathrm{UO}_{2}$ fuel[4, 5]. Additionally, by establishing a deterministic correlation between the crystallographic orientation and surface morphology, it is possible to develop a method to deduce the lattice orientation of surface grains of a $\mathrm{UO}_{2}$ sample based on its surface faceting features.

As a consequence of anisotropic surface energy, the surface faceting phenomenon occurs in a great number of crystalline materials. The surface faceting features can also be correlated with the Wulff shape of the crystal[6]. The formation mechanism for surface faceting has been investigated by both experimental and computational approaches $[7,8,9,10]$. Previously, the surface faceting of $\mathrm{UO}_{2}$ was investigated by low energy electron diffraction (LEED). However, as LEED is limited to the characterization of single crystal specimens, only those planes with low Miller indices; namely, $\{100\},\{110\}$, and $\{111\}$ planes; have been examined[11, 12, 13, 14]. As the closest packed layer, the $\{111\}$ surface of $\mathrm{UO}_{2}$ has the lowest surface energy[15, 16] and is, therefore, not subject to surface coarsening or faceting[17]. On the contrary, the $\{110\}$ surface forms a wavy structure consisting of two $\{111\}$ planes sharing a $<110>$ edge with the angle of $109.47^{\circ}$ as a result of annealing. In addition, although the majority of the $\{100\}$ surface remains smooth during annealing, hemi-octahedra (square pyramids) that contain four $\{111\}$ planes, form on the $\{100\}$ surface. These pyramid features are believed to be due to the deposition of $\mathrm{UO}_{2}$ vapor, which is generated during annealing. Only $\{111\}$ and $\{100\}$ planes are present at equilibrium, while the $\{110\}$ and higher-index planes are absent. All the edges present on equilibrium $\mathrm{UO}_{2}$ surfaces are $<110>$ type, as reported by previous studies[14]. The dominance 
of the $\{111\}$ and $\{100\}$ planes and the $<110>$ edge is also comparable to previous studies of the morphology of $\mathrm{UO}_{2}$ single crystal. Theoretical prediction gives a simple $\{111\}$ faceted octahedron[15]. Meanwhile, the experimental examination is limited to some SEM observation of voids on $\mathrm{UO}_{2}$ surfaces, which shows a $\{111\}$ faceted octahedron truncated by $\{100\}$ planes $[18$, 19]. The appearance of $\{100\}$ planes might be explained by non-equilibrium kinetics model[15] or the modification of surface energy due to hydroxidation[20, 21]. Hence, further studies are necessary to clarify the morphology of $\mathrm{UO}_{2}$ crystal. Recently, the $\{100\}$ and $\{111\}$ surfaces were characterized by scanning tunneling microscopy (STM), revealing the atomic level structure of these low-index crystallographic planes[22, 23].

All previous investigations on the surface faceting features of $\mathrm{UO}_{2}$ are limited to three low Miller index planes pesent within single crystal specimens. There is a scarity of comprehensive studies on the faceting features of surfaces with general indices. In order to develop a comprehensive understanding of the faceting behavior of polycrystalline $\mathrm{UO}_{2}$, two key properties need to be determined: the crystallographic orientation of the grains, and a quantitative description of their corresponding surface faceting features. The coarse surface of $\mathrm{UO}_{2}$ with faceting features limits the utilization of conventional techniques. For instance, although electron backscattering diffraction (EBSD) is capable of working with coarse surfaces, the faceting features may create shadows on investigated surfaces, preventing the collection of grain to grain matching maps of crystallographic orientation, especially when grains are small. However, synchrotron X-ray diffraction is barely influenced by surface roughness due to the deep penetration ability originating from its high energy and intensity, making it an ideal technique for this study. This synchrotron technique has been adopted to examine a variety of bulk materials, including advanced Fe-based alloys[24, 25, 26, 27, 28, 29] and metallic nuclear fuel materials[30, 31]. When the X-ray beam is focused to a submicron size, mapping of a micrograined specimen can be easily achieved[32]. Thus, synchrotron Laue microdiffraction[33] has the unique non-destructive capability of measuring the crystallographic orientation of grains with coarse surfaces. The surface features can be characterized by secondary electron (SE) imaging with a scanning electron microscope (SEM). Synchrotron microdiffraction and SE SEM techniques were coordinated in this study to establish 
the precise correlation between crystallographic orientation and the surface faceting features of multiple grains within a polycrystalline $\mathrm{UO}_{2}$ specimen.

\section{Experiments}

\subsection{Specimen Preparation}

The polycrystalline $\mathrm{UO}_{2}$ specimen investigated in this study was fabricated utilizing spark plasma sintering (SPS)[34]. $\mathrm{UO}_{2}$ powder was procured from International Bio-analytical Industries Inc. To reduce the particle size, the powders were ball milled for 30 minutes utilizing tungsten carbide container and milling balls. To produce bulk $\mathrm{UO}_{2}$ samples, an SPS within an industrial argon atmosphere ( $<5 \mathrm{ppm}$ oxygen) was used. The temperature was increased to $1600^{\circ} \mathrm{C}$ with a $100^{\circ} \mathrm{C} / \mathrm{min}$ ramp rate, while the pressure was increased from a $10 \mathrm{MPa}$ pre-load to $40 \mathrm{MPa}$. The sample was sintered for 5 minutes under these conditions, and then the temperature was decreased to $1500^{\circ} \mathrm{C}$ at $20^{\circ} \mathrm{C} / \mathrm{min}$ as the pressure was decreased to $10 \mathrm{MPa}$. The sample was annealed for 30 minutes under these conditions in order to relieve residual stresses induced during sintering. The power was turned off, and the sample was allowed to cool to room temperature. Further details about the ball milling and SPS procedures can be found in Ref. [34]. The sintered $\mathrm{UO}_{2}$ sample was mechanically polished down to $0.5 \mu \mathrm{m}$ roughness using diamond lapping films to produce a uniformly smooth surface finish. The sample was then thermally etched by annealing at $1500^{\circ} \mathrm{C}$ for 1 hour in a He gas environment to activate the formation of surface faceting by enhancing surface diffusion. By measuring the lattice parameter using an PANalytical X-ray diffractometer, the stoichiometry of the sample was determined according to the following equation: $a=5.4705-0.132 x[35,36]$, where $a$ is the derived lattice parameter, and $x$ is the stoichiometry parameter as in the formula $\mathrm{UO}_{2+x}$. The stoichiometry of the sample investigated in this study was found to be $\mathrm{UO}_{2.00047 \pm 0.00608}$. No distinguishable oxygen pickup was detected during the thermal etching procedure based on the lattice parameter measurement.

\subsection{Synchrotron Microdiffraction}

Synchrotron Laue microdiffraction measurements were performed at Sector 34-ID-E at the Advanced Photon Source (APS), Argonne National Laboratory (ANL). The synchrotron experiment 
setup is illustrated in Figure 1(a). The $\mathrm{UO}_{2}$ specimen was kept in a sealed container with a Kapton film window. The synchrotron white X-ray beam was focused by a Kirkpatrick-Baez (K-B) mirror system to provide a $0.6 \mu \mathrm{m} \times 0.8 \mu \mathrm{m}$ beam size for $2 \mathrm{D}$ scanning. The sample was oriented such that the sample surface was at a $45^{\circ}$ angle to the beam direction. Laue diffraction patterns were collected by a $2048 \times 20482 \mathrm{D}$ area detector at an array of points across the sample. The Laue patterns were then processed to determine the reciprocal lattice vectors so that the crystal orientation of all the scanned positions could be derived. The scanning step length in both directions was $2 \mu \mathrm{m}$. Because the grain size of the $\mathrm{UO}_{2}$ sample was $18.93 \pm 1.28 \mu \mathrm{m}$ according to the SEM images, approximately 70 data points were collected for each grain.

\subsection{Surface Analysis}

The surface morphology of the $\mathrm{UO}_{2}$ specimen was captured by a Hitachi S-3000N SEM. As the SEM only captures $2 \mathrm{D}$ projections of $3 \mathrm{D}$ surface features, the specimen was tilted to two angles $\left(0^{\circ}\right.$ and $\left.45^{\circ}\right)$ for the regions of interest, to avert the loss of information during projection, as shown in Figure 1(c). The tilting axis is the $\mathrm{x}$-axis of the specimen coordination system $\left(x^{\prime}\right)$.

Previous investigations on both surface faceting and morphology of $\mathrm{UO}_{2}$ at equilibrium showed the dominance of $\{111\}$ planes as well as the coexistence of $\{100\}$ planes. However, $\{110\}$ surfaces, and surfaces with higher Miller indices were not observed at equilibrium[14]. Therefore, the $\{111\}$ and $\{100\}$ planes are assumed to be the dominant surface facets for analysis in this study, namely, all the edges observed are assumed to be $<110>$ type.

Any orientation of a $\mathrm{UO}_{2}$ lattice can be described by three Euler angles: $\phi, \theta$ and $\psi$, representing three rotation operations, respectively. Any point in the lattice coordinate system, $P=[x, y, z]^{T}$, can be transformed into the corresponding coordinates in the specimen coordinate system, $P^{\prime}=$ $\left[x^{\prime}, y^{\prime}, z^{\prime}\right]^{T}$ through the Euler rotations described by the following expression ( $x$-convention):

$$
P^{\prime}=\left(\begin{array}{ccc}
\cos \psi & \sin \psi & 0 \\
-\sin \psi & \cos \psi & 0 \\
0 & 0 & 1
\end{array}\right)\left(\begin{array}{ccc}
1 & 0 & 0 \\
0 & \cos \theta & \sin \theta \\
0 & -\sin \theta & \cos \theta
\end{array}\right)\left(\begin{array}{ccc}
\cos \phi & \sin \phi & 0 \\
-\sin \phi & \cos \phi & 0 \\
0 & 0 & 1
\end{array}\right) P
$$


Because the specimen was also tilted to $45^{\circ}$ in SEM, the coordinate of the point in the tilted specimen coordinate system $\left(45^{\circ}\right), P^{\prime \prime}=\left[x^{\prime \prime}, y^{\prime \prime}, z^{\prime \prime}\right]^{T}$ experiences an extra rotation operation:

$$
P^{\prime \prime}=\left(\begin{array}{ccc}
1 & 0 & 0 \\
0 & \frac{1}{\sqrt{2}} & -\frac{1}{\sqrt{2}} \\
0 & \frac{1}{\sqrt{2}} & \frac{1}{\sqrt{2}}
\end{array}\right) P^{\prime}
$$

As mentioned previously, $<110>$ are assumed to be the dominant edges on the $\mathrm{UO}_{2}$ surfaces, and all six $<110>$ edges were considered in this study. These six $<110>$ edges can be defined by 5 points in the lattice coordinate system: $P_{0}=[0,0,0]^{T}, P_{1}=[-1,0,-1]^{T}, P_{2}=[1,0,-1]^{T}$, $P_{3}=[0,-1,-1]^{T}$, and $P_{4}=[0,1,-1]^{T}$. If $L_{i j}=P_{j}-P_{i}=\left[x_{j}-x_{i}, y_{j}-y_{i}, z_{j}-z_{i}\right]^{T}$ represents the direction vector of the edge defined by the two points $P_{i}$ and $P_{j}$, the six unique $<110>$ edges are respectively $L_{01}, L_{02}, L_{03}, L_{04}, L_{13}$, and $L_{14}$. In the specimen coordinate system $\left(0^{\circ}\right)$, those $L_{i j}$ become $L_{i j}^{\prime}=P_{j}^{\prime}-P_{i}^{\prime}$ from Equation 1 . At a $45^{\circ}$ tilt in the SEM, the corresponding direction vectors of the $<110>$ edges are $L_{i j}^{\prime \prime}=P_{j}^{\prime \prime}-P_{i}^{\prime \prime}$, as described in Equation 2. Because the information of the third component $\left(z_{j}^{\prime}-z_{i}^{\prime}\right.$ and $\left.z_{j}^{\prime \prime}-z_{i}^{\prime \prime}\right)$ of the edge direction vector is lost during the 2D projection in SEM imaging, the first and second components of $L_{i j}^{\prime}$ and $L_{i j}^{\prime \prime}$ determine the direction of the specific $<110>$ edges observed in SEM images. Polar coordinates were used to define the $<110>$ edges in the SEM images. The polar angles are defined as: $l_{i j}^{\prime}=\operatorname{atan} 2\left(y_{j}^{\prime}-y_{i}^{\prime}, x_{j}^{\prime}-x_{i}^{\prime}\right)$ (zero tilt) and $l_{i j}^{\prime \prime}=\operatorname{atan} 2\left(y_{j}^{\prime \prime}-y_{i}^{\prime \prime}, x_{j}^{\prime \prime}-x_{i}^{\prime \prime}\right)\left(45^{\circ}\right.$ tilt $)$, where $\operatorname{atan} 2$ is the two-argument arctangent function.

\section{Results}

\subsection{Orientation-Dependent Surface Faceting}

An approximately $250 \mu \mathrm{m} \times 400 \mu \mathrm{m}$ region of the polycrystalline $\mathrm{UO}_{2}$ specimen was selected to perform both Laue microdiffraction and SEM measurements (Figure 2). The orientation map with inverse pole figure (IPF) coloring from the Laue microdiffraction patterns was compared to the surface topography images obtained by the SEM. Grain to grain matching between the two images confirmed that both techniques captured the same region on the specimen. Based 
on the orientation information obtained by microdiffraction, the surface faceting features can be correlated to the crystallographic orientation of each individual grain.

The presence of surfaces with low Miller indices $(\{100\},\{110\}$ and $\{111\})$ that were observed in previous studies was confirmed. Due to the unique nature of this study, a much broader range of surface normal orientations were able to be observed. The unique surface features of these orientations were mapped out on the standard stereographic triangle (SST), and are described in the following paragraph. Figure 3 elucidates the change in surface morphology as the surface normal changes from $<001>$ to $<111>$, along a $<110>$ axis (on side of the SST). When the surface normal is near $<001>$, the surface is dominated by a $\{100\}$ plane with a series of hemi-octahedra made of four $\{111\}$ planes (see the blue point in Figure 3 ). The large pyramids sometimes are truncated by a $\{100\}$ plane to form a frustum. When the surface normal is slightly inclined from $<001>$, the pyramids are also inclined, and the surface becomes stepped by $\{111\}$ planes (see the green point in Figure 3). As the surface normal becomes closer to $<111>$, the fraction of $\{111\}$ planes increases. When the surface normal is close to $<111>$, the surface is dominated by a $\{111\}$ plane, with steps formed by a $\{100\}$ plane, and residual inclined pyramids or frusta(see the red point in Figure 3). When the surface normal is perfectly $<111\rangle$, it is theoretically a smooth $\{111\}$ plane.

Figure 4 shows the evolution of the surface morphology as the surface normal evolves from $<001>$ to $<101>$ along a $<010>$ axis. When the surface normal is near $<001>$, the dominant feature is still a stepped $\{100\}$ plane with $\{111\}$ square pyramids or frusta. However, as the surface normal tilts towards $<101>$, the steps are no longer dominated by one $\{111\}$ plane (as occurred when the surface normal was tilted towards $<111>$ ), but split between two $\{111\}$ planes (see the red point in Figure 4). As the surface normal approaches $<101>$, the proportion of the two $\{111\}$ planes continues to increase, as illustrated by the green and blue points in Figure 4. When the surface normal is close to $<101>$, the surface is dominated by a wavy feature containing two $\{111\}$ planes and stepped by a $\{100\}$ plane (see the orange point in Figure 4).

Figure 5 tracks the surface morphology as the surface normal changes from $<111>$ to $<101>$ along a $<10 \overline{1}>$ axis. As the surface normal tilts from $<111>$ to $<101>$, the original $\{111\}$ plane 
starts to be stepped by another $\{111\}$ plane with a $107.49^{\circ}$ angle. When the surface normal is close to $<101>$, the fractions of the two types of $\{111\}$ planes are equal, creating the characteristic wavy features of the $\{110\}$ surface. It is worth mentioning that the $\{100\}$ planes are present in the SEM images in Figure 5 because the surface normal is not exactly on the side.

After investigating the surface faceting features near the three vertexes and along the three boundaries of the SST, regions with a surface normal falling in the core area of the SST were also examined. Multiple surface normal vectors within this area were studied, some representative cases of which are shown in Figure 6. For all those cases, two $\{111\}$ planes and one $\{100\}$ plane are present with three $<110>$ edges, forming the so-called triple-plane structure, as defined in this study. The fraction of each plane type is determined by the specific position on the SST. For example, if the surface normal is relatively close to $<001>$, the $\{100\}$ plane is dominant (see the red point in Figure 6). Similarly, if the surface normal is relatively close to $<111>$, a $\{111\}$ plane is dominant (see the green point in Figure 6), and if the surface normal is relatively close to $<101>$, two $\{111\}$ planes are equally dominant to form a wavy feature, as shown by the blue point in Figure 6.

Therefore, the triple-plane structure is the main component of $\mathrm{UO}_{2}$ surfaces with general surface normals. Even the smooth $\{111\} /\{100\}$ surface and the wavy $\{110\}$ surface can be regarded as variants of the triple-plane structure, where one or two of the three planes is overwhelmingly dominant. The only exception occurs when the surface normal is close to $\langle 100\rangle$, where the $\{100\}$ plane can be elaborated by square pyramids or frusta. According to previous calculations of surface energies of the $\{111\},\{100\}$, and $\{110\}$ planes of $\mathrm{UO}_{2}[16,15]$, the $\{111\}$ plane has the lowest surface energy, whereas the $\{100\}$ plane has the highest surface energy among the three planes of lowest Miller indices. As the closest packed layer of the fluorite structure, the $\{111\}$ plane has lower surface energy compared to the $\{110\}$ plane. The wavy feature consisting of two $\{111\}$ planes, which have an angle of $109.47^{\circ}$, is preferentially formed over a smooth $\{110\}$ plane once $\gamma_{110}>\sqrt{3 / 2} \gamma_{111}$. The inequality is satisfied according to the surface energy calculations $[16,15]$, explaining the absence of $\{110\}$ planes throughout the studies on $\mathrm{UO}_{2}$ surfaces, including the present one. Due to a relatively higher surface energy, $\{100\}$ planes can 
be replaced by a square pyramid composed of four $\{111\}$ planes as long as $\gamma_{100}>\sqrt{3} \gamma_{111}$. This can be confirmed by the fact that $\{100\}$ planes are usually accompanied by a series of pyramids. However, a great fraction of $\{100\}$ planes are still smooth and free of square pyramids or frusta, showing that $\gamma_{100}<\sqrt{3} \gamma_{111}$. This controversy in surface energy anisotropy also determines whether the Wulff shape of $\mathrm{UO}_{2}$ is truncated by $\{100\}$ planes or not. According to both DFT and empirical calculation of surface energy anisotropy of $\mathrm{UO}_{2}[15,16], \gamma_{100}$ is much larger than $\sqrt{3} \gamma_{111}$. A recent STM investigation on the $\{100\}$ surface of $\mathrm{UO}_{2}$ showed that the "smooth" $\{100\}$ surface is actually covered by trenches of atomic level $\{111\}$ facets[23]. Accordingly, the effective surface energy of $\{100\}$ plane, $\gamma_{100}^{\text {eff }}$ is reduced but still exceeds $\sqrt{3} \gamma_{111}[21]$. However, the surface of $\mathrm{UO}_{2}$ can be hydroxidized. Computational results show that $\gamma_{100}^{\text {eff }}$ can be lower than $\sqrt{3} \gamma_{111}$ once their surfaces are partially or entirely hydroxidized[20, 21]. Therefore, the coexistence of faceting and smooth features on $\{100\}$ surface actually originates from the local fluctuation of surface hydroxidation. On the other hand, the triple-plane structure does always include one $\{100\}$ plane, rather than three $\{111\}$ planes, regardless to the local hydroxidation situation. This is because that the three $\{111\}$ plane structure inevitably involves an acute angle $\left(70.53^{\circ}\right)$ between planes, compromising its space efficiency in covering surfaces. All the surface energy values discussed here are also listed in Table 1.

\subsection{Determination of Orientation Based on SEM Images}

Based on the assumption of the dominance of the $<110>$ edges on $\mathrm{UO}_{2}$ surface, the surface morphology has been determined as described above. To validate this assumption, the crystallographic orientations of particular grains were derived directly based on its surface morphology, and then compared with the synchrotron-based experimental data. As all the edges on the surface are $<110>$ edges, the algorithm is based on the measured projected directions of $<110>$ edges $\left(l_{i j}^{\text {exp }}\right.$ and/or $\left.l_{i j}^{\prime \prime \text { exp }}\right)$. For a specific combination of Euler angles $(\phi, \theta$, and $\psi)$, the theoretical direction vectors of the $<110>$ edges $\left(L_{i j}^{\prime}\right.$ and $\left.L_{i j}^{\prime \prime}\right)$ can be calculated according to Equations 1 and 2 . The angles of the SEM projections of the $<110>$ edges, $l_{i j}^{\text {th }}$ and $l_{i j}^{\prime \prime h}$, can be deduced from the first two components of $L_{i j}^{\prime}$ and $L_{i j}^{\prime \prime}$. A least squares estimation was used to obtain the optimized combination of Euler angles that minimize the following expression: 


$$
\sum_{i j}\left(l_{i j}^{\prime t h}-l_{i j}^{\prime e x p}\right)^{2}+\sum_{i j}\left(l_{i j}^{\prime \prime t h}-l_{i j}^{\prime \prime e x p}\right)^{2}
$$

The summation was done over all the measurable $<110>$ edges on the SEM images. At minimum, a pair of $\langle 110\rangle$ edges at both $0^{\circ}$ and $45^{\circ}$ tilting angles are required for the least squares estimation. In this study, a mesh with $1^{\circ}$ increment for all three Euler angles was used to approach the optimized combination. The misorientation $(\delta)$ between the optimized crystallographic orientation and the microdiffraction measured crystallographic orientation was used to evaluate the accuracy of the fit. The reciprocals of the values of Equation 3 for all possible combinations of Euler angles were used to evaluate the sensitivity of the fitting. In order to visualize the sensitivity examination, the reciprocals were shown in the SST according to the surface normals determined by the Euler angles. Since multiple combinations of Euler angles may share one point on the SST, only the maxima reciprocals were illustrated. The quality of the fit was further evaluated by analyzing the condition number of the Jacobian matrix of the least squares estimation at the optimized point. According to the non-linear least squares estimation theory, the Jacobian matrix has the following components:

$$
J_{i j}=\frac{\partial F_{i}}{\partial x_{j}}
$$

where, $F_{i}$ can be any measurable $l_{i j}^{\text {th }}$ or $l_{i j}^{\prime \prime h}$, and $x_{j}$ can be $\phi, \theta$, or $\psi$. The condition number, $c$, of the Jacobian matrix $J$ is defined as the ratio between the maximum and minimum singular values of $J$. The singular values were calculated by singular value decomposition of the Jacobian matrix:

$$
\begin{array}{r}
J_{m \times n}=U_{m} \Sigma_{m \times n} V_{n}^{T} \quad(m \geq n), \\
\Sigma_{m \times n}=\left(\begin{array}{c}
S_{n} \\
0
\end{array}\right),
\end{array}
$$

where $U_{m}$ and $V_{n}$ are two unitary matrices, $S_{n}=\operatorname{diag}\left\{\sigma_{1}, \sigma_{2}, \ldots, \sigma_{n}\right\}$ with $\sigma_{1} \geq \sigma_{2} \geq \ldots \geq$ $\sigma_{n}$ is a diagonal matrix containing the singular values. Determined by the three Euler angles, 
$n=3$. Therefore, $c=\sigma_{1} / \sigma_{3}$. The number of non-zero singular values indicates the number of fitting parameters (Euler angles) that can be uniquely determined. If $\sigma_{3}$ is non-zero but small, namely, the condition number is large, the Jacobian matrix is ill-conditioned, implying that the optimization results are influenced by the errors in measurement. Otherwise, the Jacobian matrix is well-conditioned, indicating that the optimization is robust.

When the surface normal is close to $\langle 100\rangle$, due to the existence of the pyramids, all six $<110>$ edges are visible in SEM images. Therefore, no tilting is needed to determine the crystallographic orientation. The fitting result is compared with the synchrotron measured orientation in Figure 7. The sensitivity of this category of fitting is quite good according to the mapping of the reciprocal least squares. The condition number of the Jacobian matrix is as low as 12.4. The only exception occurs as the surface normal is exactly at $\langle 100>$, namely, $\theta=0$ and only $\psi+\phi$ can be uniquely determined, this also results in a zero $\sigma_{3}$, implying that only two of three Euler angles can be uniquely determined.

In most cases, the triple-plane structure dominates the surface morphology. Thus, only three of six $<110>$ edges are visible on the surface. Assuming the three observable edges are $L_{01}, L_{02}$, and $L_{04}$, SEM images from both tilting angles $\left(0^{\circ}\right.$ and $\left.45^{\circ}\right)$ were used for determining the Euler angles. Because one $\{100\}$ plane and two $\{111\}$ planes are involved, the plane types need to be assumed prior to any fitting. There are three possible combinations of plane types that fulfill the triple-plane structure requirements. All three combination are taken into consideration, and the combination that minimizes Equation 3 is regarded as the optimized solution. Figure 8 illustrates the fitting details of a typical three-plane structure case. All three possible triple-plane structures were fitted, and only one yielded a sufficiently small value of Equation 3. The misorientation between the optimized crystallographic orientation and the microdiffraction measurement is $3.5^{\circ}$ with a condition number of 4.0 , demonstrating the adequate reliability of the fitting. When the surface normal is close to $<111>$ and is near the upper left side of the SST, all three $<110>$ edges within the $\{111\}$ planes are observable (see the red point in Figure 3 as an example). With the three measurable $<110>$ edges, the orientation can be obtained from the SEM images taken at both tilting angles. 
When the surface normal is near the right side of the SST (between $<111>$ and $<101>$ ), the fraction of the $\{100\}$ plane in the triple-plane structure is marginal. As a result, only two $<110>$ edges can be measured. Fortunately, by utilizing two tilting angles, the orientation of the grain can still be deduced. Figure 9 shows the fitting method for a case where there are only two measurable $<110>$ edges. The misorientation between the fitting result and the microdiffraction measurement is only $2.9^{\circ}$, while the condition number of the Jacobian matrix is 13.6 , indicating a sound fit.

When the surface normal is along the right boundary in the SST, between $<111>$ and $<101>$, there is only one measurable $<110>$ edge in this regime, which is inadequate for the deduction of crystallographic orientation. However, the orientation of this type of grains can still be determined to be $\mathrm{a}<1 \mathrm{x} 1>(0 \leq x \leq 1)$ type orientation.

The orientation estimation situations are summarized in Figure 11. For the majority of the surface normal orientation cases, the projected directions of two or more $<110>$ edges can be precisely measured from SEM images taken at two different tilting angles. Therefore, the Euler angles of these grains can be deduced based on the knowledge of the orientation-dependent surface faceting features discussed previously. Using this method, the misorientation between the fitted crystallographic orientation and the real crystallographic orientation is usually smaller than $5^{\circ}$. However, when the surface normal is along the right boundary of the SST, the orientation cannot be determined, as only one $<110>$ edge is measurable. This validates the assumption made for surface morphology investigation. Additionally, the surface morphology of $\mathrm{UO}_{2}$ also provides an alternative method of measuring crystallographic orientation of surface grains with acceptable uncertainty only using SE images. In addition to the standard mechanical polishing procedures for SEM/EBSD investigations, this alternative method only requires extra thermal etching at an appropriate temperature for an adequate period ( 1 hour at $1500^{\circ} \mathrm{C}$ for $\left.\mathrm{UO}_{2}\right)$ in an inert gas atmosphere. This is especially meaningful for a nuclear fuel material, the characterization of which is usually restricted due to their radioactivity.

Aside from $\mathrm{UO}_{2}$, this technique might potentially be applied to other materials. Two prerequisites are required for successful adoption of this method: prominent anisotropy in surface energy and a 
highly symmetrical crystal structure. The former prerequisite ensures the preferential appearance of some characteristic surface orientations, whereas the latter one helps reduce the complexity in geometry. Thus, this method is expected to work for any cubic materials with large surface energy dependence on crystallographic orientation. On the other hand, for those materials with low-symmetry crystal structures, the determination of crystallographic orientation solely based on surface morphology could be challenging, as the number of different orientations increases greatly in the absence of symmetry reduction.

\section{Conclusions}

In this study, synchrotron Laue microdiffraction and SEM techniques were collaboratively utilized so as to establish a general correlation between the crystallographic orientation and surface faceting features of polycrystalline $\mathrm{UO}_{2}$. Sets of low-Miller-index planes were found across the sample, with a dependence upon the surface normal. A triple-plane structure, which contains one $\{100\}$ plane, two $\{111\}$ planes, and three $<110>$ edges, is the major surface faceting feature for most orientations. The dominance of this structure can be explained by the preference in thermodynamic preference for low-energy surfaces. The coexistence of $\{100\}$ and $\{111\}$ planes on $\{100\}$ surface also implies the variance of $\gamma_{100} / \gamma_{111}$, probably due to the local fluctuation of surface hydroxidation. The knowledge of the orientation-dependent surface faceting features was used to develop a method to determine the orientation of a grain based on SEM images. This technique is applicable for the vast majority of crystallographic orientations.

\section{Acknowledgments}

This work was funded by the U.S. Department of Energy (DOE)'s Nuclear Energy Advanced Modeling and Simulation (NEAMS) program and the U.S. DOE's Nuclear Energy University Program (NEUP) DE-NE0008440. This research used resources of the Advanced Photon Source, a U.S. DOE Office of Science User Facility operated for the DOE Office of Science by Argonne National Laboratory under Contract No. DE-AC-02-06CH11357 between UChicago Argonne, LLC and the U.S. Department of Energy. 


\section{References}

[1] M. Lyons, R. Boyle, J. Davies, V. Hazel, T. Rowland, Uo2 properties affecting performance, Nuclear engineering and design 21 (1972) 167-199.

[2] M. Notley, I. J. Hastings, A microstructure-dependent model for fission product gas release and swelling in uo2 fuel, Nuclear Engineering and Design 56 (1980) 163-175.

[3] M. Tucker, Grain boundary porosity and gas release in irradiated uo2, Radiation Effects 53 (1980) 251-255.

[4] T. Kutty, K. Chandrasekharan, J. Panakkal, J. Ghosh, Fracture toughness and fracture surface energy of sintered uranium dioxide fuel pellets, Journal of materials science letters 6 (1987) 260-262.

[5] K. Forsberg, A. Massih, Diffusion theory of fission gas migration in irradiated nuclear fuel uo2, Journal of nuclear materials 135 (1985) 140-148.

[6] J. W. Cahn, C. A. Handwerker, Equilibrium geometries of anisotropic surfaces and interfaces, Materials Science and Engineering: A 162 (1993) 83-95.

[7] W. Mullins, Theory of linear facet growth during thermal etching, Philos. Mag. 6 (1961) 1313-1341.

[8] E. D. Williams, N. C. Bartelt, Surface faceting and the equilibrium crystal shape, Ultramicroscopy 31 (1989) $36-48$.

[9] E. D. Williams, N. Bartelt, Thermodynamics of surface morphology, Science 251 (1991) 393-400.

[10] J. R. Heffelfinger, C. B. Carter, Mechanisms of surface faceting and coarsening, Surface science 389 (1997) 188-200.

[11] W. Ellis, R. Schwoebel, Leed from surface steps on uo 2 single crystals, Surf. Sci. 11 (1968) 82-98.

[12] W. P. Ellis, Leed studies of uo 2 ( 111) vicinal surfaces, Surface Science 45 (1974) 569-584.

[13] T. Taylor, W. Ellis, A leed study of uo 2 ( 100) vicinal surfaces, Surface Science 77 (1978) 321-336.

[14] V. Kraševec, B. Navinšek, Faceting of uo2 single crystal surfaces, physica status solidi (a) 30 (1975) 501-509.

[15] M. Abramowski, R. W. Grimes, S. Owens, Morphology of uo 2, Journal of Nuclear Materials 275 (1999) 12-18.

[16] F. N. Skomurski, R. C. Ewing, A. L. Rohl, J. D. Gale, U. Becker, Quantum mechanical vs. empirical potential modeling of uranium dioxide (uo2) surfaces:(111),(110), and (100), American Mineralogist 91 (2006) 1761-1772.

[17] S. Dudarev, M. Castell, G. Botton, S. Savrasov, C. Muggelberg, G. Briggs, A. Sutton, D. Goddard, Understanding stm images and eels spectra of oxides with strongly correlated electrons: a comparison of nickel and uranium oxides, Micron 31 (2000) 363-372.

[18] W. Van Lierde, On the surface free energy anisotropy of uo2, Journal of Materials Science 5 (1970) $527-531$.

[19] M. R. Castell, Wulff shape of microscopic voids in uo 2 crystals, Physical Review B 68 (2003) 235411.

[20] A. H. Tan, M. Abramowski, R. W. Grimes, S. Owens, Surface defect configurations on the (100) dipolar surface of uo 2, Physical Review B 72 (2005) 035457.

[21] A. H. Tan, R. W. Grimes, S. Owens, Structures of uo 2 and puo 2 surfaces with hydroxide coverage, Journal of nuclear materials 344 (2005) 13-16. 
[22] M. Castell, C. Muggelberg, G. Briggs, D. Goddard, Scanning tunneling microscopy of the uo2 (111) surface, Journal of Vacuum Science \& Technology B 14 (1996) 966-969.

[23] C. Muggelberg, M. Castell, G. Briggs, D. Goddard, An stm study of the uo 2 (001) surface, Applied surface science 142 (1999) 124-128

[24] K. Mo, Z. Zhou, Y. Miao, D. Yun, H.-M. Tung, G. Zhang, W. Chen, J. Almer, J. F. Stubbins, Synchrotron study on load partitioning between ferrite/martensite and nanoparticles of a 9cr ods steel, J. Nucl. Mater. 455 (2014) $376-381$.

[25] Y. Miao, K. Mo, Z. Zhou, X. Liu, K.-C. Lan, G. Zhang, M. K. Miller, K. A. Powers, J. Almer, J. F. Stubbins, In situ synchrotron tensile investigations on the phase responses within an oxide dispersion-strengthened (ods) 304 steel, Mater. Sci. Eng. A 625 (2015) 146-152.

[26] Y. Miao, K. Mo, Z. Zhou, X. Liu, K.-C. Lan, G. Zhang, M. K. Miller, K. A. Powers, Z.-G. Mei, J.-S. Park, et al., On the microstructure and strengthening mechanism in oxide dispersion-strengthened 316 steel: A coordinated electron microscopy, atom probe tomography and in-situ synchrotron tensile investigation, Mater. Sci. Eng. A (2015).

[27] Y. Miao, K. Mo, B. Cui, W.-Y. Chen, M. K. Miller, K. A. Powers, V. McCreary, D. Gross, J. Almer, I. M. Robertson, et al., The interfacial orientation relationship of oxide nanoparticles in a hafnium-containing oxide dispersion-strengthened austenitic stainless steel, Mater. Charact. 101 (2015) 136-143.

[28] G. Zhang, Z. Zhou, K. Mo, Y. Miao, X. Liu, J. Almer, J. F. Stubbins, The evolution of internal stress and dislocation during tensile deformation in a $9 \mathrm{cr}$ ferritic/martensitic (f/m) ods steel investigated by high-energy x-rays, Journal of Nuclear Materials 467 (2015) 50-57.

[29] G. Zhang, K. Mo, Y. Miao, X. Liu, J. Almer, Z. Zhou, J. F. Stubbins, Load partitioning between ferrite/martensite and dispersed nanoparticles of a $9 \mathrm{cr}$ ferritic/martensitic (f/m) ods steel at high temperatures, Materials Science and Engineering: A 637 (2015) 75-81.

[30] D. Brown, D. Alexander, K. Clarke, B. Clausen, M. Okuniewski, T. Sisneros, Elastic properties of rolled uranium-10wt.\% molybdenum nuclear fuel foils, Scr. Mater. 69 (2013) 666-669.

[31] Y. Miao, K. Mo, B. Ye, L. Jamison, Z.-G. Mei, J. Gan, B. Miller, J. Madden, J.-S. Park, J. Almer, et al., High-energy synchrotron study of in-pile-irradiated u-mo fuels, Scripta Materialia 114 (2016) 146-150.

[32] D. Yun, Y. Miao, R. Xu, Z. Mei, K. Mo, W. Mohamed, B. Ye, M. J. Pellin, A. M. Yacout, Characterization of high energy xe ion irradiation effects in single crystal molybdenum with depth-resolved synchrotron microbeam diffraction, Journal of Nuclear Materials 471 (2016) 272 - 279.

[33] J.-S. Chung, G. E. Ice, Automated indexing for texture and strain measurement with broad-bandpass x-ray microbeams, Journal of applied physics 86 (1999) 5249-5255.

[34] T. Yao, S. M. Scott, G. Xin, J. Lian, Tio 2 doped uo 2 fuels sintered by spark plasma sintering, Journal of Nuclear Materials 469 (2016) 251-261.

[35] K. Teske, H. Ullmann, D. Rettig, Investigation of the oxygen activity of oxide fuels and fuel-fission product systems 
by solid electrolyte techniques. part i: Qualification and limitations of the method, Journal of Nuclear Materials 116 (1983) 260-266.

[36] T. Wangle, V. Tyrpekl, M. Cologna, J. Somers, Simulated uo 2 fuel containing csi by spark plasma sintering, Journal of Nuclear Materials 466 (2015) 150-153. 
Table 1: Surface energies of $\mathrm{UO}_{2}$ surfaces of different indices.

\begin{tabular}{cccccc}
\hline$\{111\}$ & $\{100\}$ (flat) & $\{100\}$ (trench) & $\{110\}$ & Ref. & Comments \\
\hline 1.18 & 2.35 & n/a & 1.74 & {$[15]$} & Empirical potential \\
0.27 & 1.04 & n/a & 0.73 & {$[16]$} & DFT \\
1.29 & 2.85 & 2.45 & 2.04 & {$[21]$} & Empirical potential \\
0.85 & 1.54 & 1.18 & 1.21 & {$[21]$} & Empirical potential; 50\% surface hydroxidation \\
\hline
\end{tabular}


(a) 2D Detector

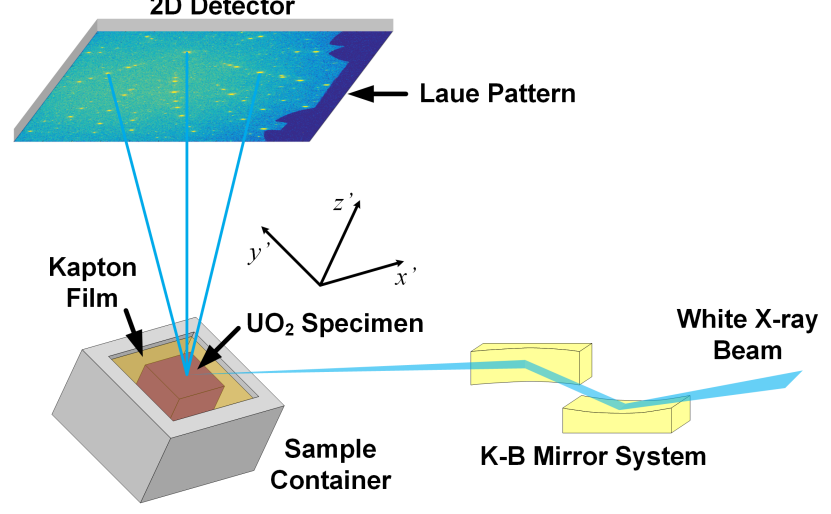

(b)
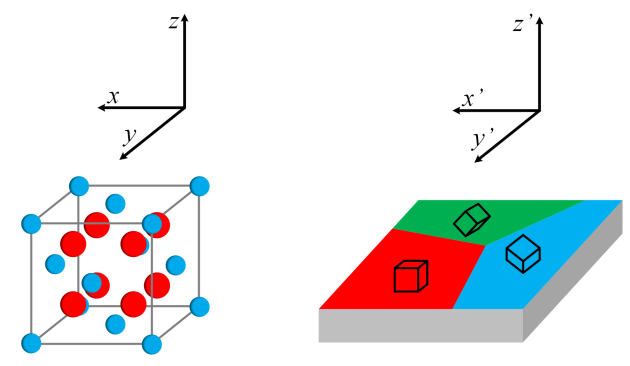

(c)

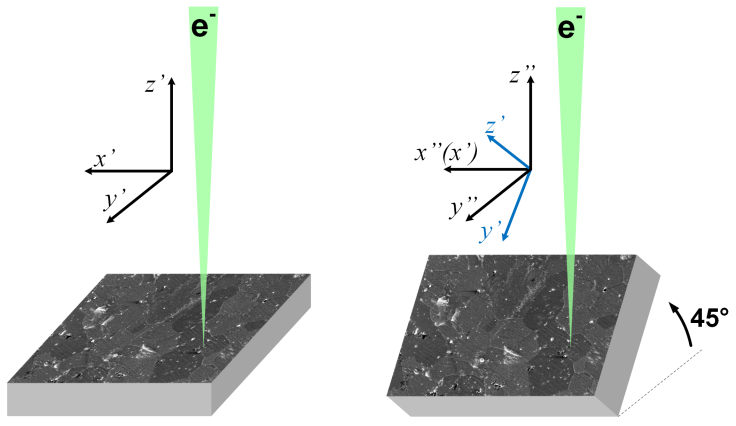

Figure 1: the experiment setups: (a) the setup of the synchrotron Laue microdiffraction experiment; (b) the relationship between the lattice coordinate system of $\mathrm{UO}_{2}$ with fluorite structure (left) and the specimen coordinate system (right): each grain in the specimen has its own lattice coordination system and corresponding Euler angles for transformation to the specimen coordinate system; (c) the tilting setup of the SEM investigations: the specimen coordinate system (') and tilted specimen coordinate system (") are shown in this figure: the $z^{\prime}$ direction is the surface normal. 


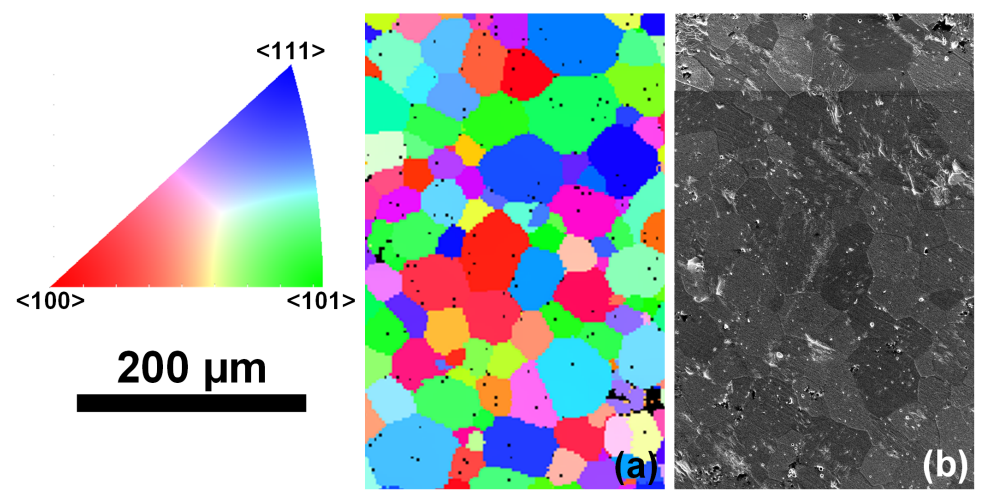

Figure 2: Same region in the $\mathrm{UO}_{2}$ specimen characterized by synchrotron Laue microdiffraction and SEM: (a) orientation map determined by Laue microdiffraction patterns with inverse pole figure (IPF) coloring. The black spots represent those positions of which crystallographic orientations failed to be indexed; (b) SEM secondary electron image.

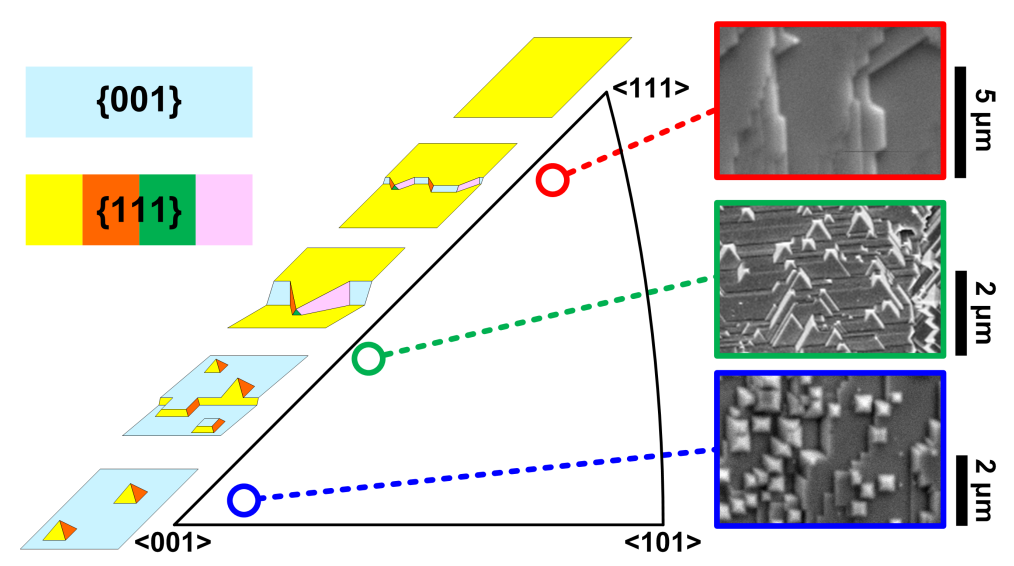

Figure 3: Surface faceting features with surface normal from $<100\rangle$ tilting to $\langle 111>$ : surface morphology was illustrated by both schematics and SEM images on the SST. 


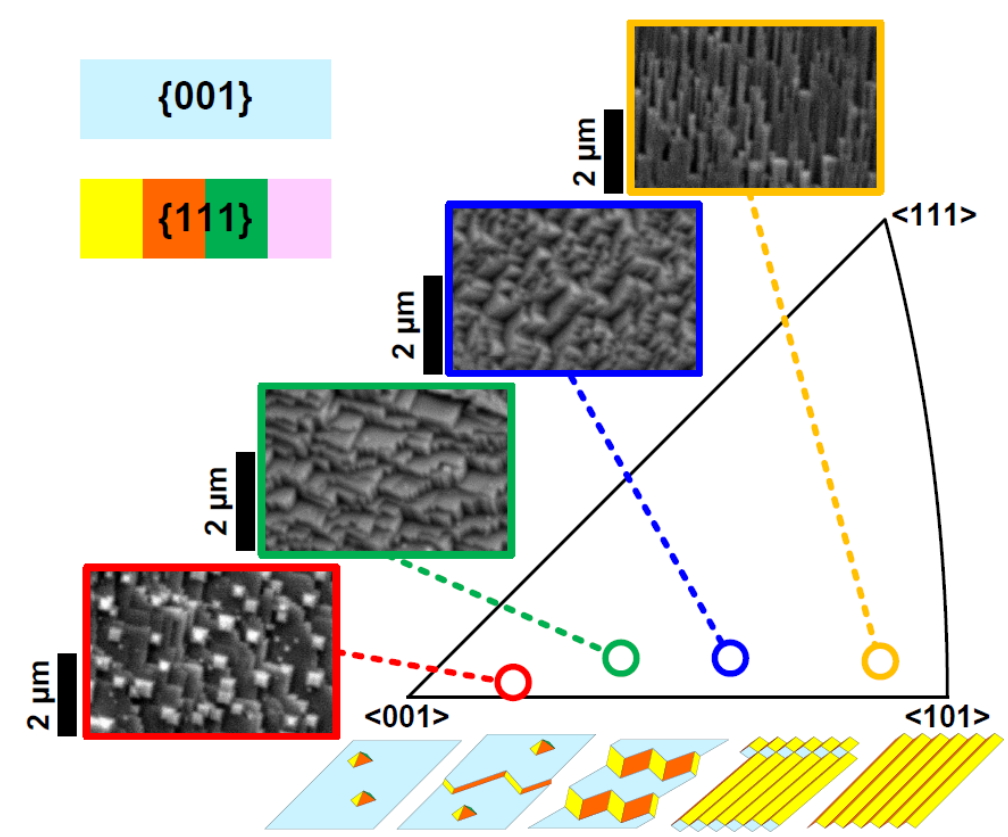

Figure 4: Surface faceting features with surface normal from $\langle 100\rangle$ tilting to $\langle 110\rangle$ : surface morphology was illustrated by both schematics and SEM images on the SST.

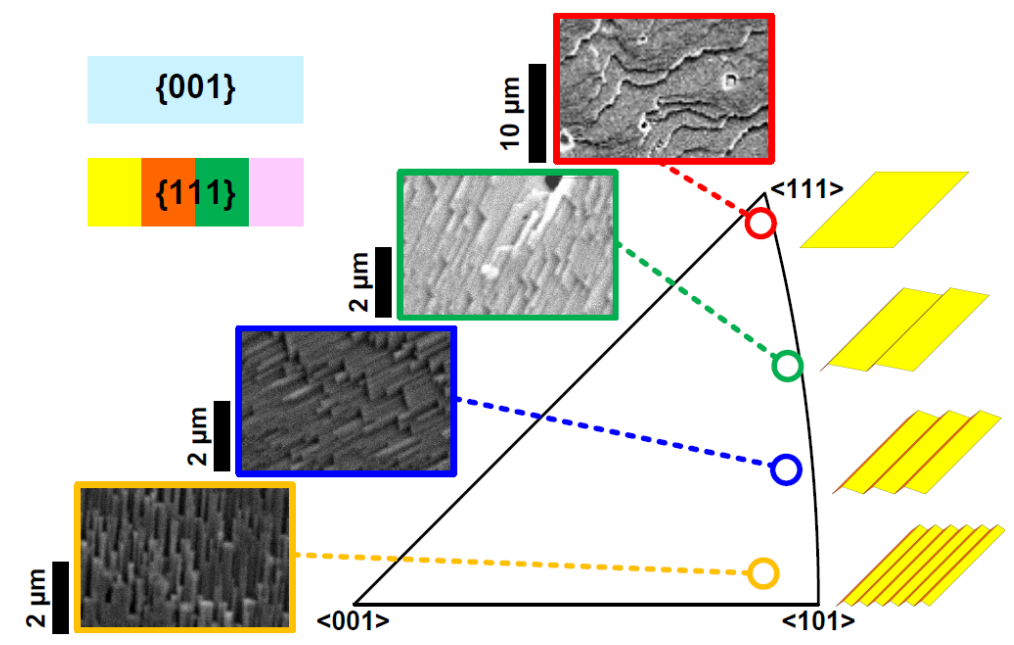

Figure 5: Surface faceting features with surface normal from $\langle 111\rangle$ tilting to $\langle 110\rangle$ : surface morphology was illustrated by both schematics and SEM images on the SST. 


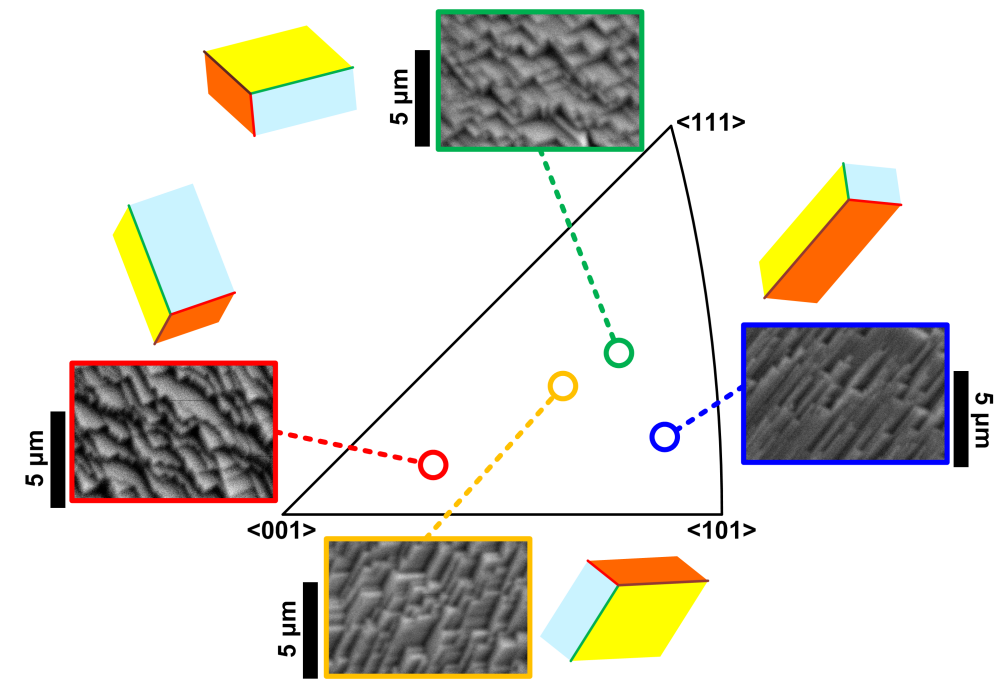

Figure 6: Surface faceting features with surface normal far away from the edges: surface morphology was illustrated by both schematics and SEM images on the SST.

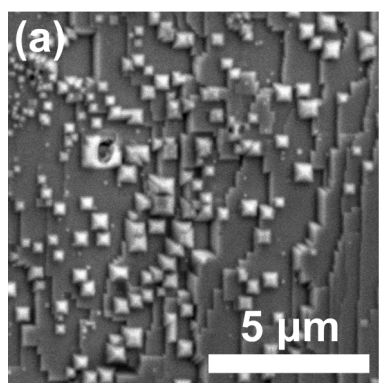

(b)

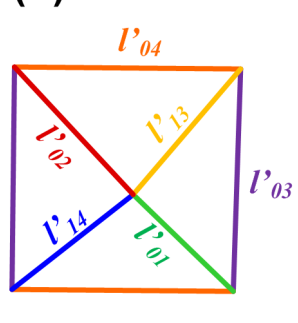

(c)

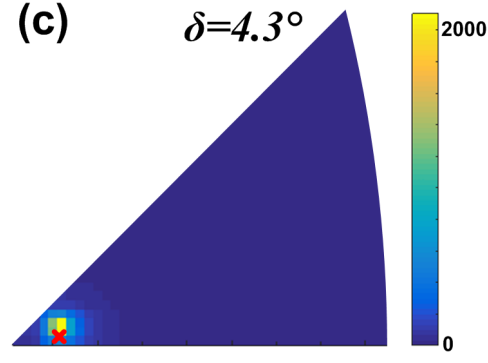

Figure 7: Fitting details of a typical grain with a surface normal near $<100>$ : (a) the SEM image of a grain with surface normal close to $\langle 100\rangle$; (b) schematic indicating the six measurable $\langle 110\rangle$ edges in (a); (c) the reciprocals of Equation 3 with surface normal vectors throughout the SST. The red cross marks the crystallographic orientation determined by synchrotron diffraction. 

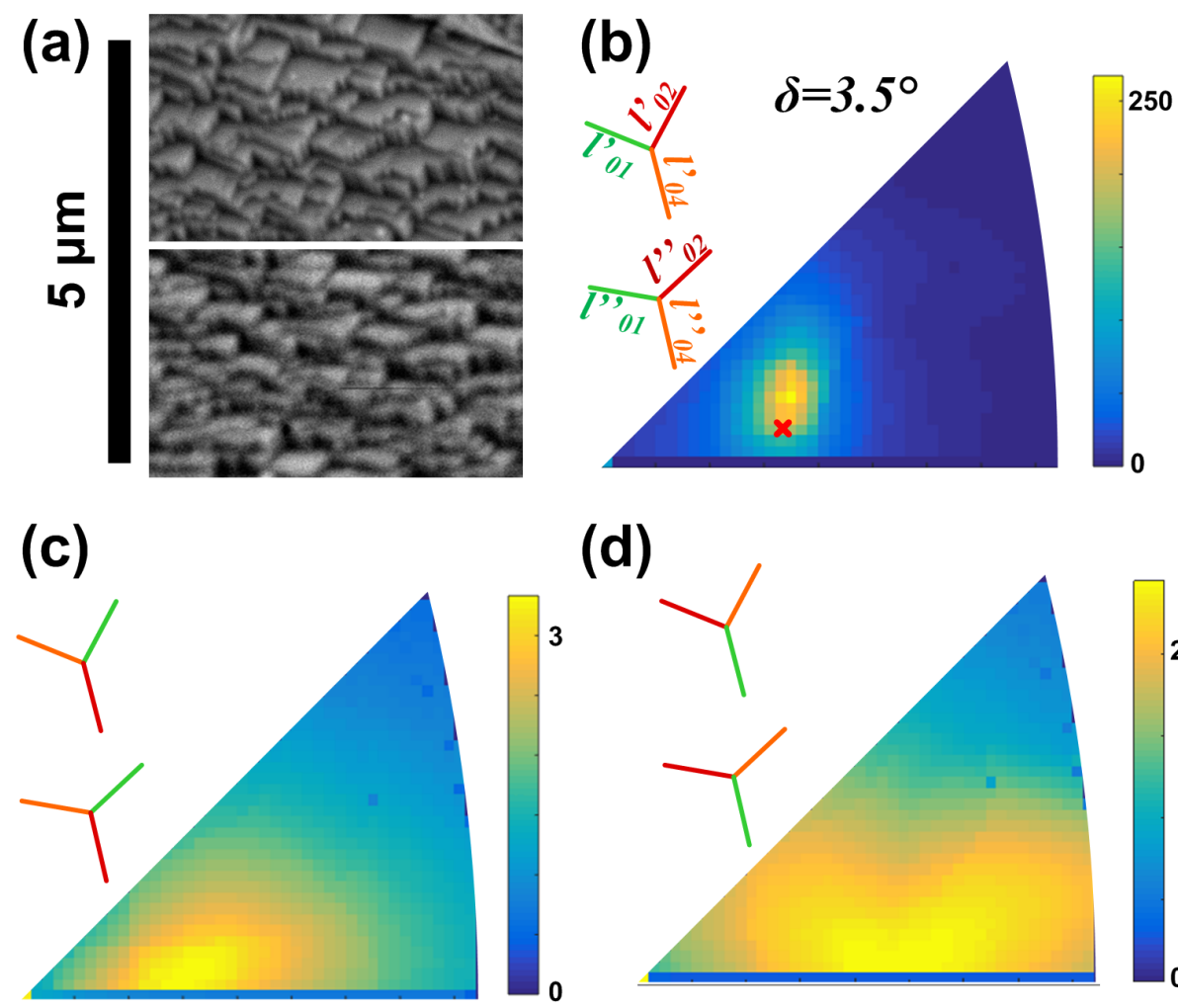

(d)

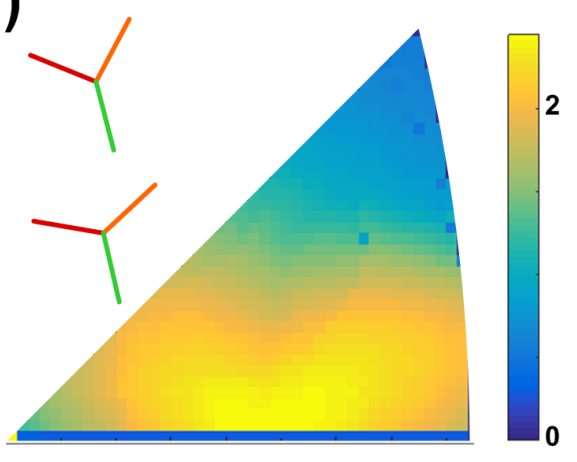

Figure 8: Fitting details of a typical three-plane feature with three measurable $<110>$ edges: (a) the SEM image of a grain with the triple-plane surface feature; (b) the correct combination of the three measurable $\langle 110\rangle$ edges and the corresponding reciprocals of Equation 3 with surface normal vectors throughout the SST. The red cross marks the crystallographic orientation determined by synchrotron diffraction; (c) and (d) the incorrect combinations of the three measurable $<110>$ edges and the corresponding reciprocals of Equation 3 with surface normal vectors throughout the SST. 

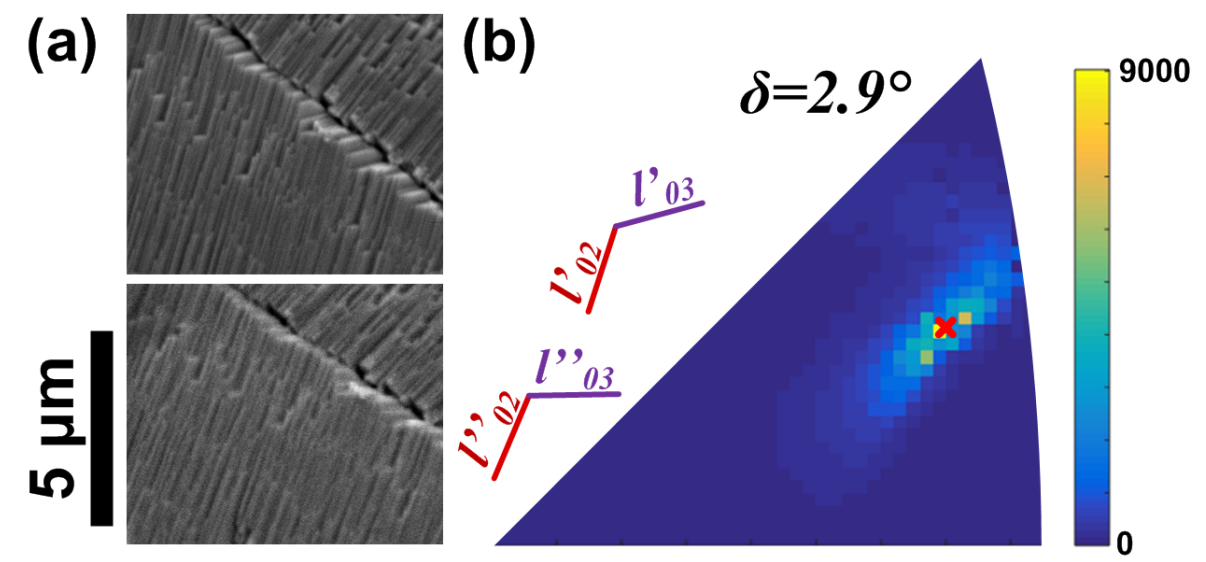

Figure 9: Fitting details of a typical three-plane feature with two measurable $<110>$ edges: (a) the SEM image of a grain with two measurable $<110>$ edges; (b)the cartoon indicating the two measurable $<110>$ edges in (a) and corresponding reciprocals of Equation 3 with surface normal vectors throughout the SST. The red cross marks the crystallographic orientation determined by synchrotron diffraction.

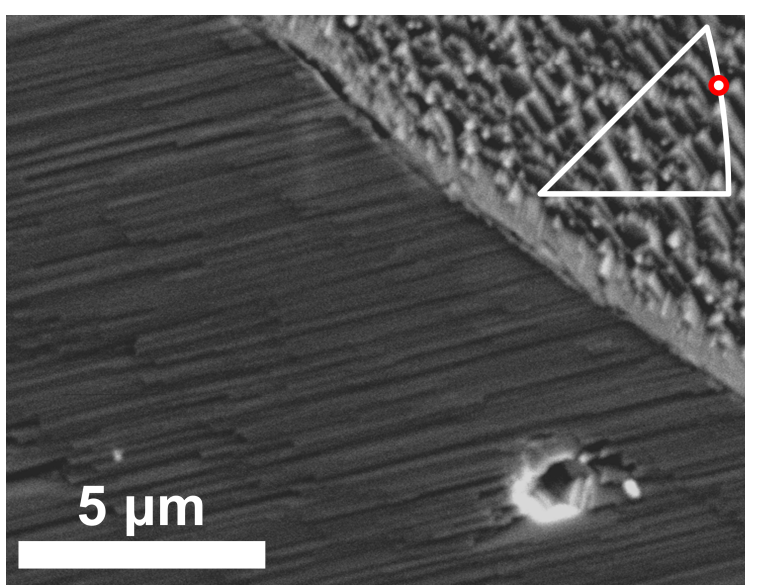

Figure 10: An SEM image of a typical case near $<1 \times 1>$ surface normal orientation, where there is only one measurable $<110>$ edge. The position of the surface normal in the SST is shown in the upper right corner. 


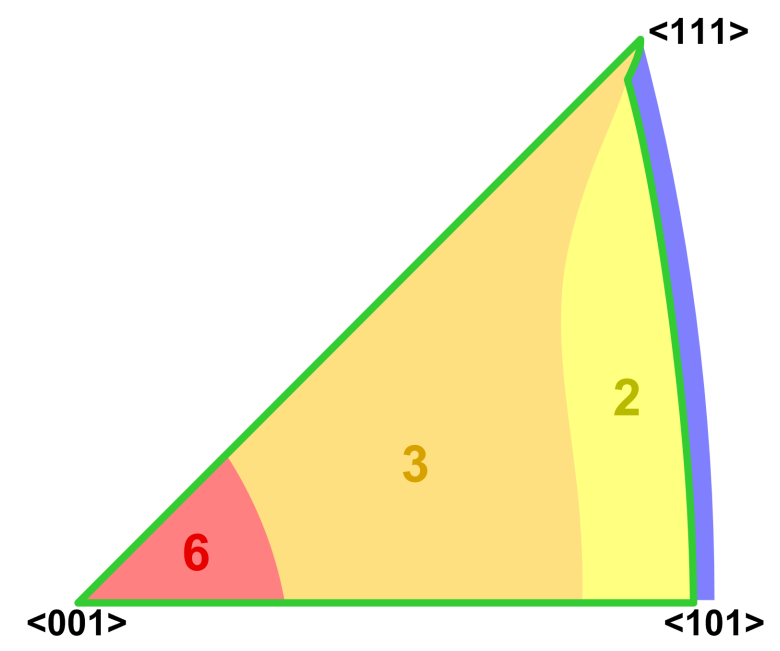

Figure 11: An SST map summarizing the possibility of determining a grain's crystallographic orientation from SEM images, the regimes outlined in green have sufficient information available from SEM images for orientation determination. The number within each regime indicates the number of measurable $<110>$ edges at a specific tilting angle. The region in blue does not have enough information from SEM images to determine the crystallographic orientation. 